(9), Sjögren Syndrome (5) and mixed connective tissue disease (5). The allO group included rheumatoid arthritis (28), psoriatic arthritis (10), spondyloarthritis (11), and JIA (8). The proportion of children born at term was lowest in patients with SLE $(79 \%)$, compared to OCTD $(91 \%)$ and allO patients $(88 \%)$. The table shows selected baseline and outcome parameters.

In early pregnancy, more patients with SLE reported low disease activity and a higher proportion was diagnosed by the rheumatologist as having mild disease than in the other diagnoses groups. During pregnancy, flares occurred rarely in SLE and OCTD patients, whereas a substantial proportion of patients in the allO group had multiple flares. However, serious complications during and after pregnancy occurred more often in SLE and OCTD patients, as well as congenital anomalies and complications. Due to the low number of events and completed pregnancies, associations to suspected risk factors couldn't be analyzed yet. Conclusions: Although most patients with SLE or OCTD had a favorable course and outcome of pregnancy, compared to patients with other inflammatory rheumatic diseases substantially more complications and adverse pregnancy outcomes occurred. The association to specific risk factors predicting adverse pregnancy outcomes could not yet be analyzed. At the time of the EULAR congress we expect at least twice as much completed pregnancies.

All patients with SLE and OCTD should be tightly screened during pregnancy with timely and adequate adjustment of drug treatments.

Acknowledgements: Rhekiss is a collaborative project of the DRFZ Berlin and the Rheumazentrum Rhein-Ruhr e.V. Düsseldorf, jointly funded by both institutions.

Disclosure of Interest: None declared

DOI: 10.1136/annrheumdis-2017-eular.6714

\section{THU0262 CHANGES IN SONOELASTOGRAPHIC CHARACTERISTICS IN SJÖGREN'S SYNDROME - A FIVE-YEAR FOLLOW UP}

B. Hofauer, M. Bas, N. Mansour, A. Knopf. Otorhinolaryngology/Head and Neck Surgery, Klinikum Rechts Der Isar, Technical Universitiy Munich, Munich, Germany

Background: The role of sonography in the evaluation of salivary gland alterations in patients with suspected Sjögren's Syndrome (SS) and its benefit with regard to the diagnosis is part of continuing investigation. Studies on sonoelastographic alterations in the salivary glands of patients with SS have been published during the last years and were able to prove significant differences compared to patients with sicca-symptoms without fulfilling the AECG classification criteria [1,2]. The available knowledge on longitudinal changes in sonoelastographic characteristics is still limited.

Objectives: Which variations of sonoelastographic salivary gland alterations can be observed during the five-year follow up of patients with primary Sjögren's Syndrome?

Methods: Patients with primary Sjögren's Syndrome (pSS) diagnosed according to the AECG classification criteria were included in this study. The EULAR SS Patient Reported Index (ESSPRI) was applied for the evaluation of patient's symptoms and the EULAR SS Disease Activity Index (ESSDAI) for systemic features. During 2011 and 2016 the sonoelastographic alterations of the salivary glands in patients with primary Sjögren's Syndrome were evaluated. Acoustic Radiation Force Impulse (ARFI) imaging (=shear wave velocity), Real Time Tissue Elastography (RTTE) and Virtual Touch Tissue Imaging (VTTI) were applied for the sonoelastographic evaluation in addition to B-Mode sonography (BMUS). Results of BMUS, RTTE and VTTI were graded with appropriate scoring systems. Results: Fifty patients diagnosed with pSS were included ( 45 female, age: 56 years $S D=16)$. In 2011 the mean ESSPRI score was $8.3(S D=4.6)$ and the mean ESSDAI score was $5.6(S D=7.5)$. Initially, the mean ARFI value of parotid glands was $2.99 \mathrm{~m} / \mathrm{s}(\mathrm{SD}=0.93)$ and the mean ARFI value of the submandibular glands was $2.15 \mathrm{~m} / \mathrm{s}(\mathrm{SD}=0.57)$. Clinical examination and sonoelastographic evaluation was repeated after five years in 2016, revealing a mean ESSPRI score of 6.1 $(S D=3.2, p=0.002)$ and a mean ESSDAl score of $4.6(S D=7.0, p<0.001)$. After five years a significant decline of ARFI values could be observed in the parotid gland $(2.33 \mathrm{~m} / \mathrm{s}, \mathrm{SD}=0.70, \mathrm{p}<0.001)$ while no significant changes of the ARFI of the submandibular gland could be observed. Results of RTTE and VTTI did not change significantly. The mean time interval between onset of first symptoms and first sonographic examination in 2011 was 57.3 months ( $S D=60.8$ ).

Conclusions: The five-year sonoelastographic follow up of salivary gland alterations in patients with pSS revealed a decline in the severity of sonoelastographic alterations of the parotid gland in ARFI imaging, indicating a certain capability for modulation of salivary gland affection in pSS.

References:

[1] Hofauer B et al. Sonoelastographic Modalities in the Evaluation of Salivary Gland Characteristics in Sjögren's Syndrome. Ultrasound Med Biol 2016;2) Knopf $A$ et al. Diagnostic utility of Acoustic Radiation Force Impulse (ARFI) imaging in primary Sjoegren's syndrome. Eur Radiol 2015.

Disclosure of Interest: None declared

DOI: 10.1136/annrheumdis-2017-eular.6436

\section{THU0263 PREVALENCE OF REMISSION AND ITS EFFECTS ON ORGAN DAMAGE AND QUALITY OF LIFE IN CHINESE PATIENTS WITH SYSTEMIC LUPUS ERYTHEMATOSUS}

C.C. Mok, L.Y. Ho, S.M. Tse, K. Chan. Medicine, Tuen Mun Hospital, HK, Hong Kong

\section{Background:}

Objectives: To study the effect of disease remission on organ damage and quality of life in Chinese patients with systemic lupus erythematosus (SLE).

Methods: Patients who fulfilled the ACR classification for SLE were studied. Their remission status at last visit was determined by the European consensus (DORIS definition): (1) Complete remission (clinical SLEDAI=0, serology inactive); and (2) Clinical remission (clinical SLEDAl=0, serology active). These two categories were further divided into those who required ongoing immunosuppressive treatment (prednisone $\leq 5 \mathrm{mg} / \mathrm{d}$ or other immunosuppressive agents) and who did not. The increase in SLE organ damage (SDI) score since 5 years prior to recruitment was compared between patients who were and were not in remission for $\geq 5$ years. Participants were randomly selected for assessment of quality of life by using both the validated version of SF36 and the LupusPRO (version 1.8) and comparison was made between those who did and did not achieve remission for $\geq 5$ years by the independent Students' t-test.

Results: 769 SLE patients were studied (92\% women; age $46.4 \pm 14.6$ years, SLE duration $12.6 \pm 8.1$ years). At last visit, clinical remission was present in $259(33.7 \%)$ patients (median 43 months) and complete remission (clinically and serologically inactive) was present in 280 (36.4\%) patients (median 51 months). Clinical/complete remission for $\geq 5$ years was achieved in $64(8.3 \%)$ and 129 $(16.8 \%)$ of the patients, respectively. $53(6.9 \%)$ patients in remission $\geq 5$ years were taken off all medications including HCQ (drug-free). Compared with patients who did not remit, those remitted $\geq 5$ years were older $(49.9 \pm 13.2$ vs $45.7 \pm 15.8$; $\mathrm{p}=0.004$ ), and had significantly lower prevalence of renal involvement, leukopenia or thrombocytopenia. Significantly fewer patients who remitted for $\geq 5$ years were maintained on prednisolone compared to others $(31 \%$ vs $68 \% ; p<0.001)$. The increase in SDI scores over the preceding 5 years was $0.17 \pm 0.53$ in patients who had complete/clinical remission off-therapy (except $\mathrm{HCQ}$ ) for $\geq 5$ years $(\mathrm{N}=88$ ), $0.25 \pm 0.51$ in those remitted for $\geq 5$ years but maintained on immunosuppressive medications $(\mathrm{N}=105), 0.41 \pm 0.84$ in those remitted for $<5$ years $(\mathrm{N}=346)$ and $0.67 \pm 1.10$ in those who did not remit $(N=230)$, respectively. The increase in SDI was statistically higher in those remitted for $>5$ years than $<5$ years $(p=0.007)$ or those who did not remit $(p<0.001)$. Logistic regression showed that patients with remission for $<5$ years or who did not remit had an increase in the risk of new damage accrual as compared to those with remission for $>5$ years (OR 2.42 [1.50-3.89]; $p<0.001$ ), adjusted for age, sex, SLE duration, SDI scores 5 years prior and the daily maintenance prednisolone dose at last visit. Among 453 patients who had QOL assessment, remission for $\geq 5$ years was associated with significantly higher higher physical component and mental component scores of the SF36 than those who did not remit. Patients with remission for $\geq 5$ years had significantly higher scores in the individual health-related domains (except cognition) of the LupusPRO than those who did not remit.

Conclusions: Durable remission can be achieved in a quarter of patients with SLE. Patients with remission for $\geq 5$ years have significantly less damage accrual and better QOL. Prolonged remission is an appropriate parameter for outcome assessment in SLE.

Disclosure of Interest: None declared

DOI: 10.1136/annrheumdis-2017-eular.3947

\section{THU0264 RESPONSIVENESS OF LUPUSPRO V1.8 AMONG CHINESE PATIENTS WITH LUPUS}

C.C. Mok ${ }^{1}$, J. Block ${ }^{2}$, M. Jolly ${ }^{2} .{ }^{1}$ Medicine, Tuen Mun Hospital, HK, Hong Kong; ${ }^{2}$ Medicine and Behavorial Sciences, Rush University Medical Center, Chicago, United States

Background: LupusPRO v1.7 is a patient reported outcome tool for patients with systemic lupus erythematosus (SLE) that has undergone psychometric validation and responsiveness studies in the US.

Objectives: To report results on responsiveness of LupusPRO among Chinese patients with SLE.

Methods: 430 patients with SLE meeting the ACR classification criteria were recruited in Hong Kong, China at a single center for this observational study. LupusPRO scores from two visits one year apart were analyzed for responsiveness and Minimal Clinically Important Difference (MCID) against patient report and physician assessed anchors of changes in health. Two patient reported anchors were used (Global change in health and item 2 of Short Form 36 form). Physician assessed anchors of change in health were disease activity (Physician global assessment-PGA, SELENA-SLEDAI) and damage (SLICC-SDI/ACR). Change in PGA of $\geq 0.3$ and SELENA-SLEDAI of $\geq 4$ in either direction was used to define worsening in disease activity. Our primary outcomes of interest were was summary $\mathrm{HRQOL}$ and $\mathrm{HRQOL}$ domains, as non HRQOL domains tend not to change over short time periods. Analysis of variance was used to compare changes in LupusPRO summary and domain scores against the anchors.

Results: Mean (SD) age of participants was 42 (14) years. Ninety five percent were women. Mean (SD) PGA, SELENA-SLEDAI and SDI at baseline were $0.5(0.5), 2.9(3.0)$ and $0.7(1.2)$ respectively. Summary HRQOL, and HRQOL 